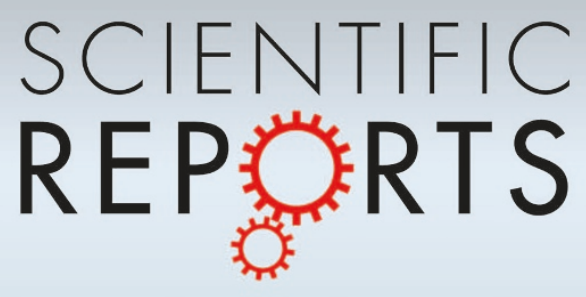

OPEN

SUBJECT AREAS:

RESPIRATORY SIGNS AND

SYMPTOMS

RESPIRATORY TRACT DISEASES

INFLAMMATION

Received

11 April 2014

Accepted

13 June 2014

Published

1 July 2014

Correspondence and requests for materials should be addressed to J.Z. (jiangcha@umich. edu) or H.Z. (zx1348@ sina.com)

\section{Dynamics of inflammation resolution and symptom recovery during AECOPD treatment}

\author{
Chun Chang' ', Zhiguo Guo', Ning Shen', Bei He', Wanzhen Yao' , Hong Zhu' \& Jiangchao Zhao
}

'Department of Respiratory Medicine, Peking University Third Hospital, Beijing, China, ${ }^{2}$ Department of Emergency Medicine, Peking University Third Hospital, Beijing, China, ${ }^{3}$ Department of Pediatrics and Communicable Disease, University of Michigan, Ann Arbor, Michigan, United States of America.

The association between increases in both systemic and airway inflammation and acute exacerbation of COPD (AECOPD) has been reported by many studies. However, relatively little is known about the dynamics of inflammation resolution and their correlations with the improvement of clinical indices during treatment. In this study, a total of 93 consecutively hospitalized patients with AECOPD were recruited. Sputum and serum inflammatory markers were measured on the day of admission before treatment (day 0 ), day 4, 7 and 14 during treatment as well as 8 weeks after discharge. Clinical indices (lung function, dyspnea and COPD assessment test (CAT) scores) were also measured at those time points. By day 4 , all airway inflammatory measures rapidly decreased and returned to baseline level. Notably, lung function and dyspnea improved to the baseline level by day 4 as well, consistent with the resolution of respiratory inflammation. However, despite the significant decrease by day 4 , systemic inflammation did not reach baseline until day 14, concordant with the decrease in CAT score. In summary, we observed a time lag between the resolution of systemic and airway inflammation, which were correlated with the improvements of different clinical indices.
C hronic obstructive pulmonary disease (COPD) is a major public health problem worldwide ${ }^{1}$. This disease is projected to rank third and fifth in terms of mortality and morbidity, respectively, in the world in $2020^{2}$. The economic and social burden caused by COPD is both substantial and increasing. According to the Global Initiative for Chronic Obstructive Pulmonary Disease (GOLD), COPD is a common preventable and treatable disease, characterized by persistent airflow limitation that is usually progressive and associated with an enhanced chronic inflammatory response in the airways and the lungs. Exacerbations and different types of comorbidities are the major factors contributing to the overall severity in individual patients ${ }^{2}$.

Acute exacerbations of COPD (AECOPD) are episodes of sustained worsening of symptoms, associated with decline of lung function, poor quality of life, and remain a major cause of mortality and morbidity ${ }^{2-6}$. The onset of AECOPD generally reflects a flare-up of extensive inflammatory processes. The link between AECOPD and increased airway and systemic inflammation has been well established ${ }^{7-10}$. However, several key questions still remain. For example, how do airway and systemic inflammation resolve during treatment with respect to the degree of resolution, time course and synchronicity? What is the relationship between the decrease in inflammation and the improvement of clinical indices?

Several studies have addressed some of the issues and advanced our understanding of the recovery of symptoms and resolution of inflammation during the treatment of AECOPD ${ }^{11-15}$. However, these studies had smaller sample sizes (20-73 patients), and either focused on only one respiratory indices (dyspnea), or only examined systemic inflammation markers or measured coarse time points. The aim of this study was to characterize changes in both airway and systemic inflammation during conventional treatment of AECOPD during a finer time course and to examine the relationship between the decrease in inflammation and the improvement of clinical indices.

\section{Results}

Characteristics of patients. A total of 93 patients were included in this study (Table 1). The median (interquartile range) age of this cohort was 67 (61-74) years old. Among these patients, 83 (89.2\%) were males and $22(22.6 \%)$ were current smokers. The median (interquartile range) values of the pack years, duration of COPD (years), and 


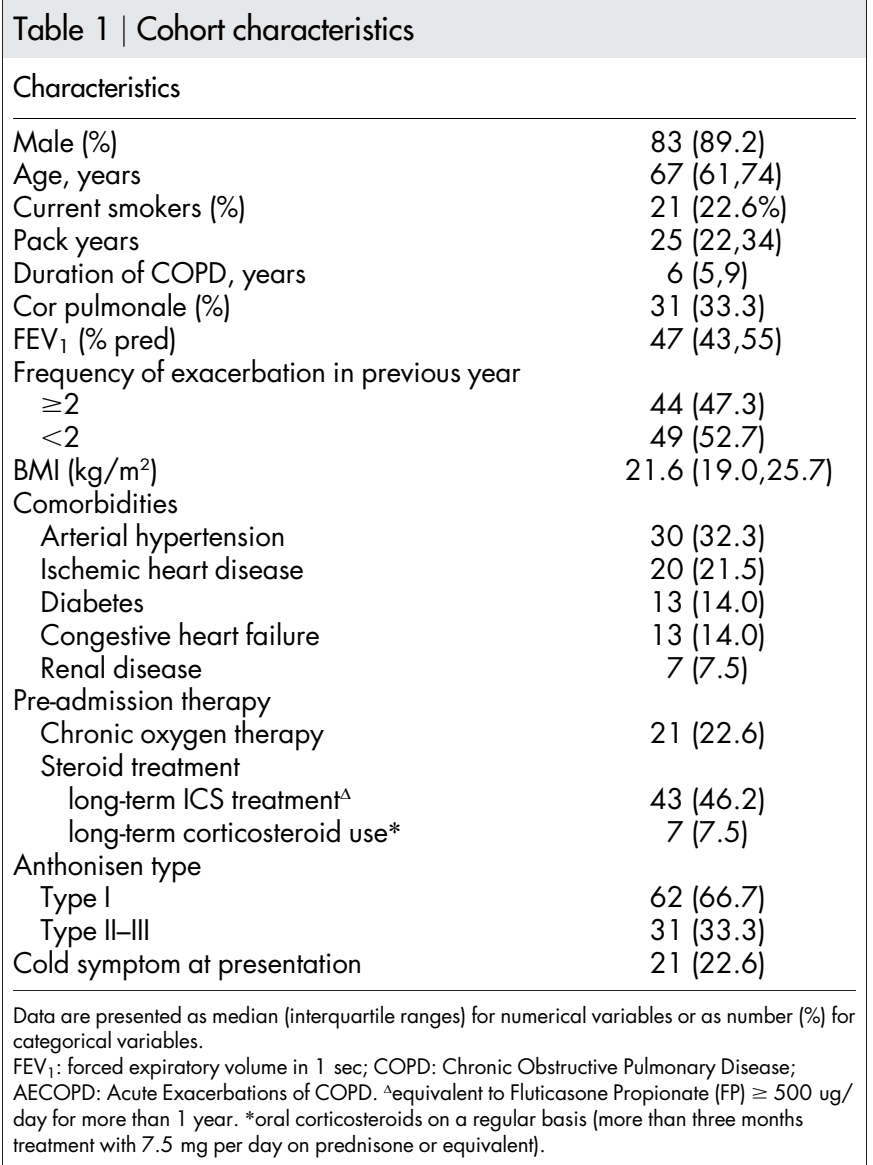

percent predicted forced expiratory volume in $1 \mathrm{~s}$ were: 25 (22-34), 6 (5-9), and 47 (43-55), respectively. Forty four patients (47.3\%) were frequent exacerbators ( $\geq 2$ per year).

Resolution of airway inflammation. Airway (sputum interleukin (IL)-8, myeloperoxidase (MPO) and neutrophils) inflammatory markers were measured before (day 0) and during conventional treatment (day 4, 7 and 14). These measures were repeated in $8 \mathrm{w}$ after discharge at the patients' subsequent baselines. Airway inflammation markers on day 0 were significantly higher than those measured in the subsequent stable state (Friedman test with Dunn's multiple comparisons, $\mathrm{P}<0.001$ for Neutropils, IL- 8 and MPO), consistent with previous studies ${ }^{4,5}$. On day 4, significant decreases in all airway inflammation markers were observed (Friedman test with Dunn's multiple comparisons, $\mathrm{P}<0.001$ for Neutropils, IL-8 and MPO). Notably, all these airway inflammation markers completely resolved, reaching a baseline level at this time point (Friedman test with Dunn's multiple comparisons, $\mathrm{P}>0.05$ for all airway inflammatory markers). No significant changes in any airway inflammation were detected at the subsequent time points compared to baseline (Friedman test with Dunn's multiple comparisons, $\mathrm{P}>0.05$ for all airway inflammatory markers, Figure $1 \mathrm{~A}-\mathrm{C}$ ).

Resolution of systemic inflammation. Systemic (serum IL-6 and Creactive protein (CRP)) inflammation markers were also measured at the same time points as the airway inflammation markers. Consistent with the airway inflammation markers and previous studies ${ }^{4,5}$, systemic inflammation markers were also significantly higher than those measured in the subsequent stable state (Friedman test with Dunn's multiple comparisons, $\mathrm{P}<0.001$ for IL-6 and CRP). Likewise, on day 4 , significant decreases in all systemic inflammation markers were also detected (Friedman test with Dunn's multiple comparisons, $\mathrm{P}<0.001$ for IL- 6 and CRP). However, despite the significant
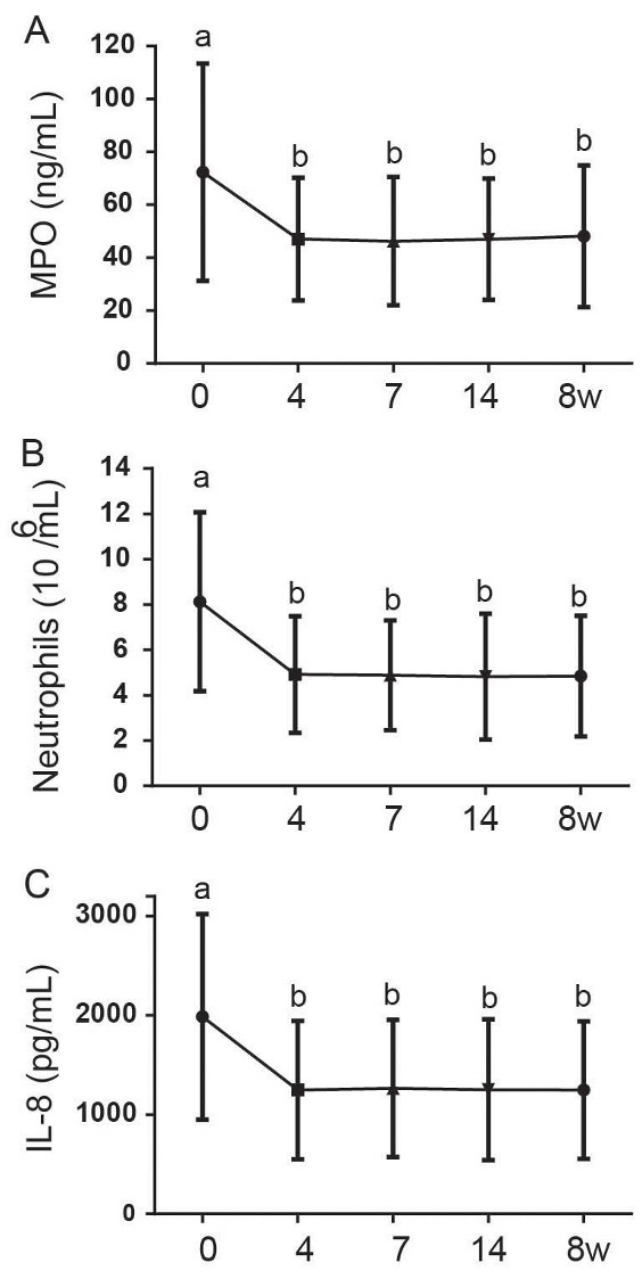

Days after admission $(0,4,7,14)$
and time after discharge $(8 \mathrm{w})$

Figure $\mathbf{1} \mid$ Resolution of airway inflammatory biomarkers including myeloperoxidase (MPO, A), neutrophils (B) and interleukin (IL)-8 (C) during the treatment of AECOPD (day 0, 4, 7 and 14) and in the subsequent baseline $(8 \mathrm{w})$.

drop on day 4, systemic inflammation did not completely resolve until day 14. Systemic inflammation markers measured on day 7 were still significantly higher than those measured on day 14 (Friedman test with Dunn's multiple comparisons, $\mathrm{P}<0.001$ for IL-6 and CRP) when they reached baseline levels ( $8 \mathrm{w}$, Figure 2A and $\mathrm{B})$.

Improvement of clinical indices. Clinical indices included lung function as measured by peak expiratory flow (PEF), modified medical research council (mMRC) dyspnea score and COPD assessment test (CAT) score ${ }^{6,7}$ and were measured in the morning before treatment on those time points.

Like the different resolution trajectories of airway and systemic inflammation, the improvement of clinical indices during the treatment fell into divergent patterns as well. The CAT scores at admission (day 0 ) were significantly higher than those measured during the subsequent stable state $(8 \mathrm{w}$ after discharge, Friedman test with Dunn's multiple comparisons, $\mathrm{P}<0.001)$. Consistent with the systemic inflammation resolution trajectory, the CAT scores, although decreased significantly at each time points (Friedman test with Dunn's multiple comparisons, $\mathrm{P}<0.001)$, did not reach baseline until day 14 (Figure 2C). 

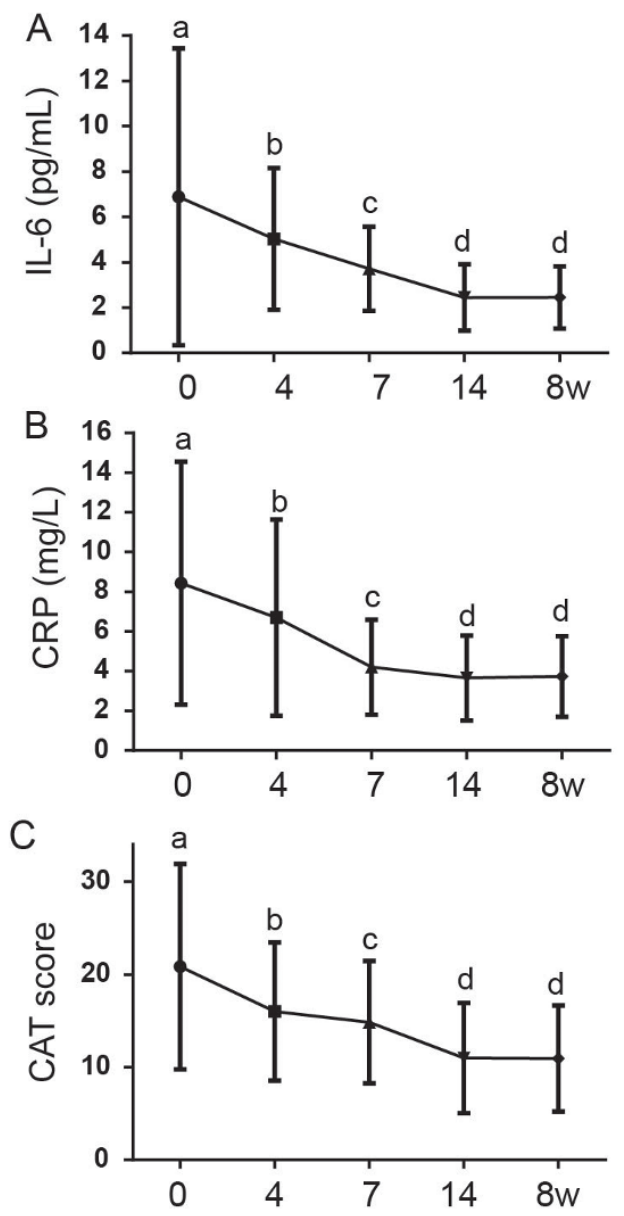

Days after admission $(0,4,7,14)$ and time after discharge $(8 \mathrm{w})$

Figure 2| Resolution of systemic inflammatory biomarkers including serum interleukin (IL)-6 (A) and C-reactive protein (CRP, B) and improvement of COPD assessment test (CAT) score (C) during the treatment of AECOPD (day 0, 4, 7 and 14) and in the subsequent baseline $(8 \mathrm{w})$.

The improvements of dyspnea (Figure 3A) and lung function (Figure 3B) were concordant with the decreases in airway inflammations. Lung function (PEF) on day 0 , although significantly lower than those in baseline (Friedman test with Dunn's multiple comparisons, $\mathrm{P}<0.001$ ), rapidly improved to the baseline level on day 4 (Figure $3 \mathrm{~B}$ ), coincident with the return to the baseline level of the airway inflammations (Figure 1) at this time point. The improvement of dyspnea symptom (mMRC) followed a path similar to the change in airway inflammation (Figure 1). On day 4, mMRC rapidly decreased to a level comparable to that of the subsequent baseline (Figure 3A).

Correlations between the changes in inflammatory markers and clinical indices. Figure 1-3 show that the changes in respiratory systems were concordant with the resolution of the respiratory markers, whereas the improvement of CAT scores was consistent with the resolution of the systemic inflammation. To test if these observations were statistically significant, we performed Mantel tests, which have been used to measure the correlations between two matrixes. We first constructed the distance matrix for each of the inflammation markers and clinical indices, measuring the euclidean distances between each time points. Therefore, the changes in inflammation and clinical indices during treatment of AECOPD were reflected in their respective distance matrixes and
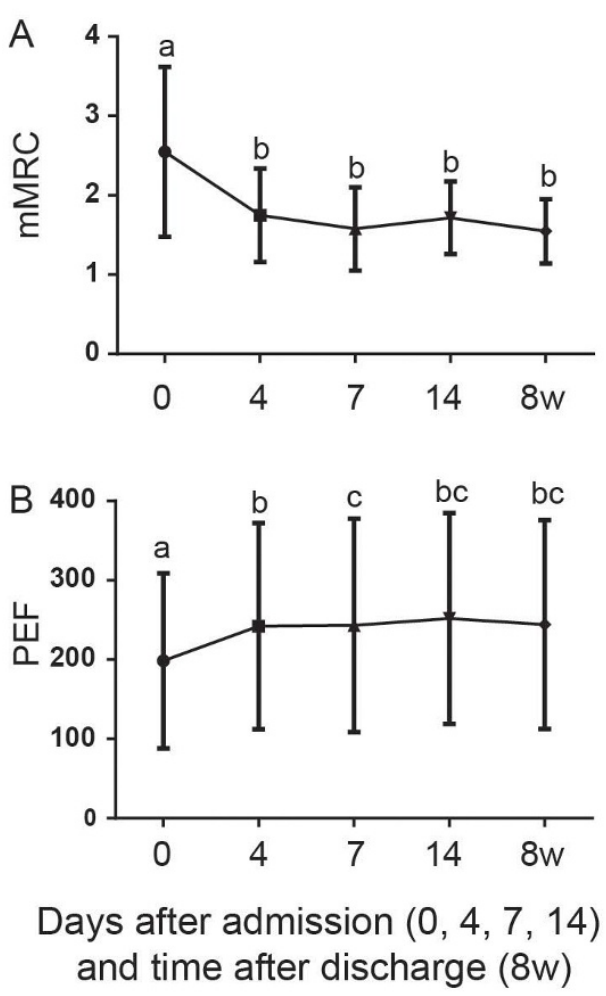

Figure $3 \mid$ Improvement of respiratory symptoms including dyspnea (modified medical research council, mMRC, A) and lung function (peak expiratory flow, PEF, B) during the treatment of AECOPD (day 0, 4, 7 and $14)$ and in the subsequent baseline $(8 \mathrm{w})$.

their pairwise relationships were tested by the Mantel test. The Mantel $\mathrm{R}$ value indicates the degree of correlation between two matrixes, with a maximum value of 1 and the Mantel $\mathrm{P}$ value shows the statistical significance.

Consistent with the observed dynamics in the plots, Mantel tests show that the improvements of respiratory symptoms were more correlated with the resolution of respiratory inflammation than the resolution of systemic inflammation. The average Mantel $\mathrm{R}$ value between PEF and respiratory inflammation markers was 0.989, which was comparable to that $(0.966)$ between dyspnea and respiratory inflammation and greater than their respective $\mathrm{R}$ values with systemic inflammation (PEF vs systemic inflammation: 0.770 , dyspnea vs systemic inflammation: 0.849 ).

Mantel test also suggested that the improvement of CAT scores was also more correlated with the resolution of the systemic inflammation than with the respiratory inflammation. The average Mantel $\mathrm{R}$ value between CAT score and systemic inflammation was 0.821 and was greater than that between CAT score and respiratory inflammation (0.776).

\section{Discussion}

Many studies have demonstrated the associations between increased inflammation and the onset of AECOPD. However, there is only very limited information regarding the dynamics of inflammation resolution and the improvement of clinical indices. In this study, we investigated the resolution trajectories of both airway and systemic inflammation markers in a large cohort of patients $(n=93)$ during the treatment of AECOPD in a finer time course. We also examined the improvement of clinical indices including lung function, dyspnea and CAT scores during the treatment of AECOPD and their relationships with the resolution of inflammations.

Due to the significant effect of AECOPD on disease progression and mortality, effective treatment of AECOPD is necessary to min- 
imize the impact of current AECOPD and to prevent the development to future exacerbations. Although a traditional 10-14 days corticosteroids treatment is recommended by GOLD guidelines ${ }^{2}$, the optimal treatment duration is unknown. Leuppi et al compared the effects of a short-term (5 days) and conventional (14 days) glucocorticoid therapy on clinical outcomes ${ }^{16}$. They found that while the short-term treatment with systemic glucocorticoids significantly reduced glucocorticoids exposure this treatment regimen was noninferior to the conventional treatment with respect to reexacerbation in 6 months. These authors also observed rapid improvement in lung function, dyspnea, quality of life and selfassessed overall performance within 5 days.

To compare the short-term and conventional treatment of AECOPD was not the objective of this study. By following the conventional treatment regimen recommended by GOLD guidelines to treat AECOPD, we detected rapid improvement in lung function and dyspnea within 4 days of treatment. The improvement in respiratory symptoms was concordant with, and was probably due to, the rapid resolution of airway inflammation biomarkers. CAT is a validated simple instrument to assess the overall health status ${ }^{17,18}$. The improvement of CAT scores was consistent with the resolution of systemic inflammatory biomarkers. Although the improvement of CAT scores and resolution of systemic biomarkers did not reach baseline until 14 days, significant changes were still observed within 4 days.

The complete recovery of respiratory symptoms and resolution of airway inflammatory biomarkers and significant improvement of CAT scores and resolution of systemic inflammatory biomarkers are consistent with the discoveries by Leuppi and collegues, supporting a short-term treatment strategy of AECOPD. However, the importance of the extended treatment on the subsequent improvement of CAT and resolution of systemic inflammatory biomarkers is unclear. CRP, one of the inflammatory biomarkers, is associated with a worse adverse outcome and severe $\mathrm{AECOPD}^{19-21}$. If the extended treatment is necessary for a complete recovery of CRP, the conventional treatment regimen is then still required.

Interleukin (IL)- 6 and IL- 8 have been used widely as biomarkers of systemic and airway inflammation, respectively. Although significant changes in IL- 6 and IL- 8 concentrations were detected longitudinally following treatment of AECOPD, no significant differences in either IL- 6 or IL- 8 concentrations were observed between patients with respect to GOLD stage (ANOVA, P $>0.05$ ) and long-term ICS treatment (student $t$ test, $\mathrm{P}>0.05$ ), very likely due to the fact that most patients $(92.5 \%)$ in this study have severe COPD (GOLD stage $\geq 3$ ). Multivariate analysis with a larger sample size and wider range of COPD severity phenotypes is desired to discern the correlations between interleukins and individual clinical measures (e.g. GOLD stage and ICS treatment).

In summary, our study shows that airway inflammation resolved completely after 4 days of treatment of AECOPD. The resolution of airway inflammation was accompanied by rapid improvement of lung function and dyspnea. Although lagging behind, systemic inflammation also resolved completely by day 14 , with a synchronous improvement of CAT score.

\section{Methods}

Ethics statement. Written informed consents were obtained from all patients. The Ethics Committee of Peking University Health Science Center approved the study protocol (IRB00001052-07095).

All experiments were performed in accordance with relevant guidelines and regulations.

Patient enrollment. A prospective study was performed between April 1, 2009 and September 30, 2011 by including consecutive patients with AECOPD admitted to a tertiary hospital center in Beijing, China. For those admitted more than once during the study period, only the first admissions were included in the analysis. The patient inclusion criteria were: COPD diagnosis and presence of exacerbation according to the Global Initiative of Chronic Obstructive Lung Disease definition (GOLD) ${ }^{2}$. Exclusion criteria were: asthma, bronchiectasis, pneumonia, cancer, sleep apnea syndrome, or other forms of active lung disease; hospitalization for reasons other than COPD exacerbation including acute coronary syndrome, congestive heart failure; need for intubation or admission to ICU; length of stay (LOS) shorter than $1 \mathrm{~d}$ or longer than $30 \mathrm{~d}$; long-term oral corticosteroids (CS) therapy (more than three months treatment with $7.5 \mathrm{mg}$ per day on prednisone or equivalent); and systemic CS therapy for exacerbation for more than $48 \mathrm{~h}$ before presentation.

Patients who had adverse clinical outcomes were also excluded. The adverse clinical outcomes (death from any cause in hospital or within 8 weeks of discharge, need for mechanical ventilation after the second hospital day, recurrent exacerbations within 8 weeks of discharge) of the patients were collected during follow up. A recurrent exacerbation was defined in the present study as a second exacerbation, fulfilling the present criteria, occurring within 8 weeks of the discharge. Symptoms from the first exacerbation must have recovered to pre-exacerbation levels.

Treatment of AECOPD. Patients were treated according to a standard protocol with nebulized salbutamol, ipratropium bromide, and systemic prednisolone (with an initial dose of 30 to $40 \mathrm{mg}$ daily). The duration of systemic prednisolone was about 2 weeks. Antibiotics were administered if bacterial infection is suspected (patientreported sputum purulence) and adjusted according to antimicrobial susceptibilities.

Mechanical ventilation was instituted by the treating physician, for indications such as respiratory arrest, deterioration in level of consciousness, and increasing partial pressure of arterial carbon dioxide despite maximal pharmacological treatment. Non-invasive ventilation was used initially whenever possible and indicated Decisions regarding admission or transfer to ICU were made by the treating unit.

Clinical variables. Variables including demographic data, spirometric values on stable condition, comorbid conditions, continuing management, smoking habits, exacerbation frequency in the previous year and respiratory symptoms were collected on the day of admission. Anthonisen type of AECOPD was determined according to the symptoms before treatment ${ }^{22}$. Exacerbation frequency in the previous year was based on the number of exacerbations the patient recalled for the year before recruitment. The COPD assessment test $(\mathrm{CAT})^{16,17}$, peak expiratory flow (PEF), and dyspnea were collected on the day of admission before treatment (day 0), during treatment (day 4, 7, and 14) and baseline ( $8 \mathrm{w}$ after discharge, stable status). PEF was measured with a mini-Wright peak flow meter (Clement Clarke, Harlow, UK) in triplicate in the morning before treatment, with the highest value recorded. Dyspnea was assessed using the 5-grade Medical Research Council dyspnea scale in the morning before treatment ${ }^{23}$.

Sample collection and measurements. Sputum and serum samples were collected on the day of admission (before treatment), during treatment (day 4, 7 and 14) and subsequent baseline ( $8 \mathrm{w}$ after discharge). Spontaneous (97\%) and induced (3\%) sputa were collected, processed within $2 \mathrm{~h}$ of collection and divided into two aliquots. One sputum aliquot was processed with phosphate buffered saline $e^{24,25}$. Cytospins were prepared and cell-free supernatant was collected and stored in aliquots at $-80^{\circ} \mathrm{C}$ pending analyses of soluble mediators. Differential cells were counted on May Grünwald Giemsa stained cytospins in a blinded fashion. Peripheral venous blood $(7 \mathrm{~mL})$ was collected into a Vacutainer tube (BD Diagnostics, NJ, USA) and centrifuged at $6716 \mathrm{G}$ for $10 \mathrm{~min}$ at $4^{\circ} \mathrm{C}$. Serum was then separated and stored at $-80^{\circ} \mathrm{C}$ till further analysis.

Serum interleukin (IL)-6 and sputum IL-8 and myeloperoxidase (MPO) were quantified using commercial sandwich ELISA kits (R\&D Systems, Abingdon, UK). Serum high sensitivity C-reactive protein (hs-CRP) was measured by a latex agglutination test, using an Olympus AU5400 automatic biochemical analyzer. All the samples from each patient were measured in the same assay to reduce inter-assay variability. The limit of detection was: $0.7 \mathrm{pg} / \mathrm{mL}$ for serum IL-6, $7.5 \mathrm{pg} / \mathrm{mL}$ for sputum IL-8, $0.062 \mathrm{ng} / \mathrm{ml}$ for sputum MPO, and $0.1 \mathrm{mg} / \mathrm{L}$ for serum hs-CRP, respectively.

Statistical analysis. Statistical analysis was performed using GraphPad Prism 6 (GraphPad Software, Inc, La Jolla, CA, USA). Categorical and numerical variables were presented as $\mathrm{n}(\%)$ and median (interquartile ranges), respectively. Differences in clinical indices, airway and inflammation markers between time points (matched repeated measures) were examined using nonparametric Friedman test with Dunn's correction. Mantel test was performed by using the R package vegan with 10000 permutations and the Pearson correlation setting. Results were considered statistically significant at the $5 \%$ level.

1. Bozinovski, S. et al. Serum amyloid a is a biomarker of acute exacerbations of chronic obstructive pulmonary disease. Am J Respir Crit Care Med 177, 269-278 (2008).

2. Vestbo, J. et al. Global strategy for the diagnosis, management, and prevention of chronic obstructive pulmonary disease: GOLD executive summary. Am J Respir Crit Care Med 187, 347-365 (2013)

3. MacIntyre, N. \& Huang, Y. C. Acute exacerbations and respiratory failure in chronic obstructive pulmonary disease. Proc Am Thorac Soc 5, 530-535 (2008)

4. Anzueto, A., Sethi, S. \& Martinez, F. J. Exacerbations of chronic obstructive pulmonary disease. Proc Am Thorac Soc 4, 554-564 (2007).

5. Donaldson, G. C., Seemungal, T. A. R., Bhowmik, A. \& Wedzicha, J. A Relationship between exacerbation frequency and lung function decline in chronic obstructive pulmonary disease. Thorax 57, 847-852 (2002). 
6. Seemungal, T. A. et al. Effect of exacerbation on quality of life in patients with chronic obstructive pulmonary disease. Am J Respir Crit Care Med 157, 1418-1422 (1998).

7. Hurst, J. R. et al. Use of plasma biomarkers at exacerbation of chronic obstructive pulmonary disease. Am J Resp Crit Care 174, 867-874 (2006).

8. Hurst, J. R., Perera, W. R., Wilkinson, T. M., Donaldson, G. C. \& Wedzicha, J. A Systemic and upper and lower airway inflammation at exacerbation of chronic obstructive pulmonary disease. Am J Respir Crit Care Med 173, 71-78 (2006).

9. Drost, E. M. et al. Oxidative stress and airway inflammation in severe exacerbations of COPD. Thorax 60, 293-300 (2005).

10. Papi, A. et al. Infections and airway inflammation in chronic obstructive pulmonary disease severe exacerbations. Am J Respir Crit Care Med 173, 1114-1121 (2006).

11. Pinto-Plata, V. M. et al. Systemic cytokines, clinical and physiological changes in patients hospitalized for exacerbation of COPD. Chest 131, 37-43 (2007).

12. Parker, C. M., Voduc, N., Aaron, S. D., Webb, K. A. \& O’Donnell, D. E. Physiological changes during symptom recovery from moderate exacerbations of COPD. Eur Respir J 26, 420-428 (2005).

13. Stevenson, N. J., Walker, P. P., Costello, R. W. \& Calverley, P. M. Lung mechanics and dyspnea during exacerbations of chronic obstructive pulmonary disease. $\mathrm{Am} \mathrm{J}$ Respir Crit Care Med 172, 1510-1516 (2005).

14. Perera, W. R. et al. Inflammatory changes, recovery and recurrence at COPD exacerbation. Eur Respir J 29, 527-534 (2007)

15. Koutsokera, A. et al. Clinical, functional and biochemical changes during recovery from COPD exacerbations. Respir Med 103, 919-926 (2009).

16. Leuppi, J. D. et al. Short-term vs conventional glucocorticoid therapy in acute exacerbations of chronic obstructive pulmonary disease: the REDUCE randomized clinical trial. JAMA 309, 2223-2231 (2013).

17. Jones, P. W. et al. Development and first validation of the COPD Assessment Test. Eur Respir J 34, 648-654 (2009).

18. Mackay, A. J. et al. Usefulness of the Chronic Obstructive Pulmonary Disease Assessment Test to evaluate severity of COPD exacerbations. Am J Respir Crit Care Med 185, 1218-1224 (2012).

19. Stolz, D. et al. Copeptin, C-reactive protein, and procalcitonin as prognostic biomarkers in acute exacerbation of COPD. Chest 131, 1058-1067 (2007).

20. Antonescu-Turcu, A. L. \& Tomic, R. C-reactive protein and copeptin: prognostic predictors in chronic obstructive pulmonary disease exacerbations. Curr Opin Pulm Med 15, 120-125 (2009).

21. Ruiz-Gonzalez, A. et al. C-reactive protein and other predictors of poor outcome in patients hospitalized with exacerbations of chronic obstructive pulmonary disease. Respirology 13, 1028-1033 (2008)
22. Anthonisen, N. R. et al. Antibiotic therapy in exacerbations of chronic obstructive pulmonary disease. Ann Intern Med 106, 196-204 (1987).

23. Bestall, J. C. et al. Usefulness of the Medical Research Council (MRC) dyspnoea scale as a measure of disability in patients with chronic obstructive pulmonary disease. Thorax 54, 581-586 (1999).

24. Efthimiadis, A., Pizzichini, M. M., Pizzichini, E., Dolovich, J. \& Hargreave, F. E. Induced sputum cell and fluid-phase indices of inflammation: comparison of treatment with dithiothreitol vs phosphate-buffered saline. Eur RespirJ 10, 1336-1340 (1997).

25. Louis, R. et al. The effect of processing on inflammatory markers in induced sputum. Eur RespirJ 13, 660-667 (1999).

\section{Acknowledgments}

This study was supported by Special fund of Chinese Medical Association for the scientific research of chronic respiratory tract diseases (07010440052).

\section{Author contributions}

C.C.H.Z. and W.Y. designed the project. C.C., Z.G. H.Z., N.S., B.H. and W.Y. collected data, C.C. H.Z. and J.Z. analyzed data, C.C., H.Z. and J.Z. wrote the paper.

\section{Additional information}

Competing financial interests: The authors declare no competing financial interests. How to cite this article: Chang, C. et al. Dynamics of inflammation resolution and symptom recovery during AECOPD treatment. Sci. Rep. 4, 5516; DOI:10.1038/srep05516 (2014).

This work is licensed under a Creative Commons Attribution-NonCommercialNoDerivs 4.0 International License. The images or other third party material in this article are included in the article's Creative Commons license, unless indicated otherwise in the credit line; if the material is not included under the Creative Commons license, users will need to obtain permission from the license holder in order to reproduce the material. To view a copy of this license, visit http:// creativecommons.org/licenses/by-nc-nd/4.0/ 